\title{
Prevalence of Carriage of Extended-Spectrum Beta- Lactamase-Producing Enterobacteriaceae Among Pregnant Women in the Primary Health Care Center and Hospital Setting in Indonesia
}

\author{
Rochmanah Oktaviani \\ Universita Airlangga \\ Miratul Hasanah \\ Universita Airlangga \\ Wahyu Setyarini \\ Universita Airlangga \\ Hari Parathon \\ Universita Airlangga \\ Koichi Kitagawa \\ Kobe University \\ Noriko Nakanishi \\ Kobe Insitute of Health \\ Ryohei Nomoto \\ Kobe Institute of Health

\section{Kayo Osawa} \\ Kobe Tokiwa University \\ Shohiro Kinoshita \\ Kobe University \\ Itaru Hirai \\ Ryukyu University \\ Toshiro Shirakawa ( $\sim$ toshiro@med.kobe-u.ac.jp ) \\ K Kuntaman \\ Universita Airlangga
}

\section{Research article}

Keywords: ESBL, pregnant woman, faecal carriage, phylogenetic group, MLST, PFGE, Indonesia

Posted Date: July 1st, 2020

DOI: https://doi.org/10.21203/rs.3.rs-38906/v1

License: (1) This work is licensed under a Creative Commons Attribution 4.0 International License. Read Full License 


\section{Abstract}

Background: The incidence of healthy individuals carrying Extended-Spectrum Beta-Lactamase producing Enterobacteriaceae (ESBL-E), especially E. coli (ESBL-EC) and Klebsiella pneumoniae (ESBL-KP), is increasing worldwide. ESBL-E causes early or late onset of neonatal sepsis, resulting in increased morbidity and mortality rates. Although maternal-neonatal transmissions of ESBL-E have been reported in several countries, the prevalence of ESBL-E carriage among pregnant women in Indonesia is not clear. In the present study, we compared the prevalence of carriage of ESBL-E among pregnant women in a primary health care center (PHC) versus two hospitals in Indonesia and identified the phenotypic and genotypic characteristics of the isolated ESBL-E strains.

Methods: We collected rectal swab samples from 200 pregnant women who visited a PHC (101 women) or were admitted to Dr. Soetomo Referral Hospital or Airlangga University Hospital (99 women) in Surabaya, Indonesia from July to October 2018. The samples were cultivated on MacConkey Agar plates supplemented with cefotaxime $2 \mathrm{mg} / \mathrm{L}$, at $37^{\circ} \mathrm{C}$ overnight. The isolated strains were identified by Bruker MALDI Biotyper ${ }^{\mathrm{O}}$ System, phenotypic detection of ESBL was performed by the combination disk method, and antibiotic susceptibility was tested by the disk diffusion method. In addition, ESBL gene was identified by PCR and DNA sequencing and molecular epidemiological studies were performed by PFGE.

Results: ESBL-E strains were isolated from 25 (rate of fecal carriage; 24.8\%) pregnant women who visited the PHC and 49 (49.5\%) pregnant women who were admitted to the hospitals. The rate of ESBL-E carriage of pregnant women in the hospitals was significantly higher than that in the PHC. Among the 74 isolated ESBL-E strains, ESBL-EC was most frequently isolated (62 strains), followed by ESBL-KP (12 strains). In addition, bla CTX-M15 was the most frequent ESBL gene type of the isolated ESBL-E strains.

Conclusion: Our results revealed the high prevalence of ESBL-E carriage in pregnant women, especially those who were admitted to the hospitals. CTX-M15 ESBL-EC was the most frequent type of ESBL-E in the pregnant women in our study. Continuous surveillance for ESBL-E carriage in pregnant women is strongly recommended to reduce the incidence of neonatal sepsis in Indonesia.

\section{Background}

The prevalence of nosocomial and community-acquired infections of Extended Spectrum $\beta$-Lactamase (ESBL) producing Enterobacteriaceae (ESBL-E), especially Escherichia coli (ESBL-EC) and Klebsiella pneumoniae (ESBL-KP), is increasing worldwide (1-5). A study in the three tertiary referral hospitals in East and Central Java, Indonesia, found that ESBL-EC and ESBL-KP were the most frequently isolated ESBL-E, followed by ESBL-Enterobacter (6). Nation-wide surveillance of ESBL-EC and -KP among clinical isolates from the eight tertiary referral hospitals in Indonesia in 2016 showed the unexpectedly high prevalence of ESBL-producing strains in the clinical isolates of Enterobacteriaceae, up to $61.8 \%$ for ESBL-EC and 58\% for ESBL-KP, retrospectively (7). In addition, studies of ESBL-E carriage in a healthy community in Surabaya Metropolitan City, Indonesia also revealed a high prevalence of faecal carriage of ESBL-E: $79 \%$ in healthy persons in dairy cow farms (8), 56\% in the students at Faculty of Medicine, Universitas Airlangga (9), and $14 \%$ in neonatal (less than four weeks old) babies and 37\% in post-neonatal (1-2 months old) babies (10).

ESBL-E causes early or late onset neonatal sepsis, resulting in increased neonatal morbidity and mortality (11-13). Previously, Denkel and colleagues (14) investigated the prevalence of colonization with ESBL-E and methicillin-resistant Staphylococcus aureus (MRSA) in very low birth weight (VLBW; $1500 \mathrm{~g}$ ) infants and their mothers. Of 209 VLBW infants tested, $5.7 \%$ were colonized with ESBL-E and 2.3\% were colonized with MRSA. In addition, an ESBL-E positive mother was identified as a risk factor for colonization of VLBW infants with ESBL-E, whereas an MRSA-positive mother was not a risk factor for MRSA colonization in VLBW infants. Likewise, maternal-neonatal transmissions of ESBL-E have been reported 
in several countries $(13,15)$. In Indonesia, despite the high prevalence of nosocomial and community ESBL-E carriage, the prevalence among pregnant women is not clear.

Since the 2000's, the community prevalence of ESBL-E is increasing globally (4, 16-19). Surprisingly, a systemic review and meta-analysis of the prevalence of ESBL-E carriage among pregnant and post-partum women in Africa revealed that the prevalence of maternal carriage of ESBL-E in the community exceeded that in hospitals (22\% versus $14 \%)(20,21)$. In the present study, we investigated the prevalence of ESBL-E carriage among 200 pregnant women who visited a primary health care center (PHC, 101 women) or were admitted to the hospitals (99 women) in Surabaya, Indonesia from July to October 2018, and identified the phenotypic and genotypic characteristics of the isolated ESBL-E strains.

\section{Methods}

\section{Study design and setting}

This cross-sectional observational study was carried out from July to October 2018 in Surabaya, Indonesia (22). The subjects of study consisted of 101 pregnant women who visited the Jagir Primary Health Center (PHC) and had not been hospitalized within a year, and 99 pregnant women who were hospitalized at Dr. Soetomo Hospital and Airlangga University Hospital for more than two days. A history of antibacterial medication was collected by a questionnaire. Written informed consents were obtained from all participants, and the study was approved by the Ethical Committees from the Dr. Soetomo Hospital (No. 0353/KEPK/VI/2018) and Airlangga University Hospital (No. 193/KEH/2018).

\section{Isolation and identification of bacterial strains}

Rectal swab samples were taken using Amies transport media and the samples were immediately cultured on MacConkey selective media supplemented with $2 \mu \mathrm{g} / \mathrm{ml}$ cefotaxime at $37^{\circ} \mathrm{C}$, overnight. Suspected single colonies were sub-cultured in blood agar at $37^{\circ} \mathrm{C}$, overnight. The bacterial isolates were identified using a MALDI Biotyper (Bruker Daltonics K.K., Yokohama, Japan).

\section{Antimicrobial Susceptibility Testing and ESBL Screening}

A Kirby-Bauer disc diffusion test was performed using Muller-Hinton agar plates for the antimicrobial susceptibility test according to the Clinical and Laboratory Standards Institute (CLSI) recommendations (23) for the following 16 antibiotics: ceftazidime (CAZ), cefotaxime (CTX), cefepime (FEP), ampicillin (AMP), imipenem (IPM), meropenem (MEPM), amikacin (AMK), minocycline (MI), tetracycline (TE), Trimethoprim-Sulfamethoxazole/co-trimoxazole (ST), nalidixic acid (NA), ciprofloxacine (CPFX), cefmetazole (CMZ), flomoxef (FMOX), aztreonam (AZT), and piperacillintazobactam (TZP) (24). The susceptibilities for colistin (CL) and tigecycline (TGC) were determined by E-test (bioMerieux, Marcy-l'Étoile, France). The European Committee on Antimicrobial Susceptibility Testing (EUCAST) criteria were used for $\mathrm{CL}$ and TGC of Enterobacteriacea (25). ESBL screening was initially performed with the CLSI confirmatory test, using both cefotaxime (CTX/30 mg) and ceftazidime (CAZ/30 mg) disks alone and in combination with clavulanic acid (CA/10 mg) (Eiken Chemical, Tokyo, Japan). The test was considered positive when the diameter of the growth-inhibitory zone around either the CTX or the CAZ disk in combination with CA increased by $\geq 5 \mathrm{~mm}$ compared to the growthinhibitory zone around the disk containing CTX or CAZ alone (23).

\section{Detection and typing of ESBL-encoding genes and phylogenetic typing of ESBL-EC}

The DNA template was obtained using the illustra ${ }^{\text {TM }}$ bacterial genomicPrep Mini Spin Kit (GE Healthcare Japan, Tokyo, Japan). ESBL-encoding genes bla $a_{\mathrm{CTX}-\mathrm{M}-1}, b / a_{\mathrm{CTX}-\mathrm{M}-2}, b / a_{\mathrm{CTX}-\mathrm{M}-9}, b / a_{\mathrm{CTX}-\mathrm{M}-14}, b / a_{\mathrm{CTX}-\mathrm{M}-15}, b / a_{\mathrm{TEM}}$, and bla $a_{\mathrm{SHV}}$ were detected by PCR assay using the primers listed in Table 1, as previously described (9). The PCR positive samples were 
further examined for DNA sequencing to identify the type of bla-gene. The DNA sequencing of the PCR products was carried out by Eurofins Genomics (Tokyo, Japan) after purification of the PCR products by the QIAquick PCR Purification Kit (Qiagen, Hilden, Germany). The DNA alignments of ESBL-encoding genes were examined by the BLAST program (https://blast.ncbi.nlm.nih.gov/Blast.cgi). The isolates of ESBL-EC were classified into phylogenetic group A, B1, B2 or D by using multiple PCR detecting chuA, yjaA and TspE4.C2 as previously described $(9,26,27)$.

\section{Pulsed-Field Gel Electrophoresis (PFGE)}

PFGE analysis was performed for the ESBL-EC harbouring the bla $a_{\mathrm{CTX}-\mathrm{M}-15}$ gene. Preparation of total genomic DNA for PFGE was performed as follows: One loop $(1 \mu \mathrm{L})$ colonies from an overnight culture were suspended in $150 \mu \mathrm{L}$ TE buffer (10 mmol/liter Tris-HCl, pH 8.0, 1 mmol/liter EDTA). Chromosomal DNA was prepared in solid agarose plugs by mixing $150 \mu \mathrm{L}$ bacterial cell suspension with an equal volume of $1 \%$ low-melting-point agarose (SeaKem® Gold Agarose, Lonza Rockland, Inc., Rockland, ME) and then incubated overnight at $50^{\circ} \mathrm{C}$ in lysis buffer $(50 \mathrm{mmol} /$ liter Tris- $\mathrm{HCl}, \mathrm{pH} 8.0$, $50 \mathrm{mmol} /$ liter EDTA, 1\% laurylsarcosine, $1 \mathrm{mg}$ proteinase $\mathrm{K} / \mathrm{ml}$ ). Thin slices of plug were washed three times for $20 \mathrm{~min}$ in sterile distilled water and twice for $20 \mathrm{~min}$ in TE buffer. The plugs were digested overnight with $30 \mathrm{U}$ of $\mathrm{Xbal}$ (TaKaRa, Shiga, Japan) restriction enzyme according to the manufacturer's instructions (28). PFGE was performed with the GenePath System (Bio-Rad Laboratories Inc., Hercules, Calif., USA) using 1\% SeaKem® Gold Agarose (Lonza Rockland, Inc., Rockland, ME) in 2 liters of 0.5X Tris-buffered EDTA running buffer. The electrophoretic conditions were as follows: initial switch time, $5.3 \mathrm{~s}$; final switch time, $49.9 \mathrm{~s}$; run time, $20 \mathrm{~h}$; voltage, $6 \mathrm{~V} / \mathrm{cm}$; buffer temperature, $14{ }^{\circ} \mathrm{C}$. Lambda Ladders (Promega, Madison, WI, USA) were used as molecular weight standards. A dendrogram was generated using GelJ ver2 (29) calculated using the unweighted pair group average (UPGMA) and the Jaccard coefficients. Strains were defined as having a clonal relationship if they possessed a similarity higher than $90 \%$ to the PFGE profiles (30).

\section{Results}

ESBL-E carriage of pregnant women in hospitals versus PHC

In this study, rectal swab samples were collected from a total of 200 pregnant women (PHC: 101; hospital, 99) from July to October 2018 in Surabaya, Indonesia. Table 2 summarizes the characteristics and ESBL-E carriage rates in the study population. The rates for pregnant women who were older than 35 years and had a history of antibiotics use were significantly higher in the hospital group than in the PHC group $(p<0.01)$. ESBL-E strains were isolated from 25 (rate of faecal carriage; 24.8\%) pregnant women in the PHC group and 49 (49.5\%) pregnant women in the hospital group. ESBLEC strains were isolated from 20 (19.8\%) pregnant women in PHC and 42 (42.4\%) pregnant women in hospital. The rates of ESBL-E and ESBL-EC carriage of pregnant women in hospital were significantly higher than those in the $\mathrm{PHC}(p<$ 0.01). Among the 74 isolated ESBL-E strains, ESBL-EC was most frequently isolated (62 strains, carriage rate: $31.0 \%$ ), followed by ESBL-KP (12 strains, carriage rate: $6.0 \%)$.

Antimicrobial susceptibility of ESBL-E isolates

Antimicrobial susceptibility testing was performed for a total of 74 isolates of ESBL-E. The rates of drug-resistant strains are shown in Fig. 1. All isolates tested were resistant to AMP, and sensitive to carbapenems (IPM and MEPM), AMK and TGC. Only ESBL-EC and KP in the hospitals showed resistance to MI and TZP (EC 27.9\% and KP 14.3\% to MI, EC 27.9\% and KP 42.9\% to TZP). Among all strains tested, only one ESBL-EC isolate in hospital was resistant to CL. As for the susceptibilities of ESBL-EC strains against TE, ST, NA, CPFX and AZT, higher numbers of hospital strains showed resistance than PHC strains, whereas higher numbers of ESBL-EC in PHC strains showed resistance to FMOX and CMZ than hospital strains.

Identification of ESBL gene type 
In the 62 isolates of ESBL-EC and 12 isolates of ESBL-KP, bla in $19(30.6 \%)$ of ESBL-EC isolates and $6(50 \%)$ of ESBL-KP isolates (Table 3). Furthermore, the bla $a_{C T X-M-15}$ gene was detected in 11 (55\%) strains of ESBL-EC in PHC. This rate was significantly higher than the rate of $19 \%$ ESBL-EC in the hospitals $(p<0.01)$. As well as the bla $a_{\mathrm{CTX}-\mathrm{M}-15}$ gene, the bla $a_{\mathrm{CTX}-\mathrm{M}-1}, b / a_{\mathrm{CTX}-\mathrm{M}-14}, b / a_{\mathrm{CTX}-\mathrm{M}-27}, b / a_{\mathrm{CTX}-\mathrm{M}-55}$ and $b / a_{\mathrm{TEM}-1}$ genes were also identified in this study (Table 3).

Phylogenetic typing of ESBL-EC isolates

Phylogenetic types of 62 isolates of ESBL-EC were determined by multiple PCR. The results of phylogenetic typing showed that the 20 isolates of ESBL-EC in PHC were A (35\%), B1 (20\%), B2 (25\%) and D (20\%), and the 42 isolates of ESBL-EC in hospitals were A (28.6\%), B1 (28.6\%), B2 (14.3\%) and D (28.6\%). Table 4.

Xbal-PFGE banding patterns of ESBL-EC isolates harbouring the bla ${ }_{\text {CTX-M15 }}$ gene

PFGE analysis was performed to evaluate the clonal relatedness of ESBL-EC isolates harbouring the b/a $a_{\mathrm{CTX}-\mathrm{M}-15}$ gene in the PHC and the hospital (Fig. 2). Both PHC and hospital ESBL-EC isolates with the bla $\mathrm{CTX}-\mathrm{M}-15$ gene showed a variety of PFGE patterns. The only two strains (Strain Code ESBL-25 \& 45) showed a clonal relationship with a similarity higher than $90 \%$, Fig. 2), and the two strains were isolated from the pregnant women in PHC.

\section{Discussion}

Since the first community-acquired ESBL-E was reported in the late 1990s, the community carriage rate of ESBL-E has continuously increased worldwide $(16,31,32)$. Reported ESBL-E community carriage rates have increased more rapidly in some regions such as Southeast Asia, Eastern Mediterranean, and Western Pacific during the 2000s compared to other regions. Recent studies from these regions often reported an ESBL-E community carriage rate around $50 \%$ (16). In the present study, ESBL-E strains were isolated from 74 out of 200 (37\%) pregnant women in PHC and hospital settings (Table 2). Other studies in different countries have reported lower rates of ESBL-E carriage in pregnant women compared to this study, for example 2.9\% (26 out of 901) in Norway in 2012 (13), 8.6\% (18 out of 209) in Germany in 2012-2013 (14), 31.7\% (32 out of 101) in Nigeria in 2014 (33), and 18.6\% (66 out of 356) in Madagascar in 2014 (21). The high prevalence of ESBL-E carriage in pregnant women in this study is probably associated with the high prevalence of ESBLE carriage in communities in Indonesia (8-10).

The use of antibiotics is considered as the primary cause of the spread of antimicrobial resistance, and it has been reported that global consumption of antibiotics increased by $65 \%$ from 2000 to 2015 . At present, there is a strict demand for proper use of antibiotics in the medical field, and the consumption of antibiotics in developed countries tends to be suppressed. In emerging countries, it continues to increase rapidly (34). In the present study ESBL-E strains were more frequently isolated from pregnant women in hospitals $(49.5 \%)$ than pregnant women in the PHC setting $(24.8 \%)(p<0.01$, Table 2). Consistent with this finding, the rate of antibiotics use in pregnant women in hospitals (41.4\%) was significantly higher rather than the pregnant women in PHC (19.8\%) $(p<0.01$, Table 2). Antimicrobial susceptibility testing showed that all 74 ESBL-E strains were sensitive to carbapenems, including IPM, MEPM, and also sensitive to AMK and TGC. However, ESBL-EC strains isolated from hospitals were more resistant to MI, TE, NA, CL, ST, AZT and TZP compared to ESBL-EC strains isolated from the PHC (Fig. 1). On the other hand, ESBL-EC strains isolated from the PHC showed higher resistance compared to hospitals against $\mathrm{FMOX}$ and $\mathrm{CMZ}$, but these resistance rates were still comparatively low (Fig. 1).

When the emergence of ESBL-E strains began in hospitals in the 1980s, the strains were mostly Klebsiella spp. and Enterobacter spp. which contained bla $a_{T E M}$ or bla ${ }_{S H V}$ genes $(35,36)$. In contrast, community-acquired ESBL-E strains 
emerging after the 1900 s were mostly E. coli harboring the bla $C T X-M E S B L$ gene (37). In the present study isolated ESBL-E strains were mostly ESBL-EC (62 out of 74 ESBL-E strains), and 32 of the 62 ESBL-EC strains harbored the b/a $a_{C T X-M} E S B L$ gene (Table 3). In the $32 \mathrm{ESBL}-\mathrm{EC}$ strains harboring the bla $a_{\mathrm{CTX}-\mathrm{M}}$ gene, bla $a_{\mathrm{CTX}-\mathrm{M}-15}$ was the most frequently identified ESBL genotype, followed by b/a $a_{\mathrm{CTX}-\mathrm{M}-55}$, bla $a_{\mathrm{CTX}-\mathrm{M}-14,}$, bla $a_{\mathrm{CTX}-\mathrm{M}-1}$ and bla $a_{\mathrm{CTX}-\mathrm{M}-27}$ respectively (Table 3 ). In addition, the $b / a_{\mathrm{CTX}-\mathrm{M}-15}$ genotype in ESBL-EC was more frequently identified in pregnant women in PHC (55\%) than in hospitals (19\%). Consistent with these findings, the literature review by Bevan et. al. (38) reported that CTX-M15 has been the most common ESBL genotype in Southeast Asia as well as most other parts of the world in the past two decades. The review also mentioned the decline of CTX-M2 and the emergence of CTX-M27, which is a single-nucleotide variant of CTX-M14 (38). In the present study, we detected the bla ${ }_{C T X-M 27}$ gene in 2 strains of ESBL-EC and one strain of ESBL-KP, but we detected no bla ${ }_{C T X-M 2}$ in any ESBL-E strains.

In our previous study (9), we isolated 82 ESBL-E strains, including 75 ESBL-EC and 7 ESBL-KP strains, from 79 (56.0\%) out of 141 stool samples from medical students in Surabaya, Indonesia. In that study, b/a $a_{\mathrm{CTX}-\mathrm{M}-15}$ was the most common (44\%) genotype in ESBL-EC and the ESBL-EC phylogenetic groups were A (37.3\%), B1 (28.0\%), B2 (1.3\%) and D (33.3\%) (9). In the present study, we observed a similar diverse distribution of phylogenetic groups in ESBL-EC: A (19 strains, 30.6\%), B1 (16 strains, 25.8\%), B2 (11 strains, 17.7\%) and D (16 strains, 25.8\%) (Table 4), with more frequent B2 group findings (17.7\% versus $1.3 \%$ ). In the Xbal-PFGE banding patterns of ESBL-EC isolates harbouring bla CTX-M-15 $_{\text {gene, }}$ both PHC and hospital ESBL-EC isolates showed a variety of PFGE patterns and a clonal spread of ESBL-EC was merely observed (Fig. 2). In general, clonal spread of ESBL-E in the community is rare, according to previous studies including our medical student study $(9,39)$.

\section{Conclusion}

In conclusion, we investigated the prevalence of ESBL-E carriage in pregnant women in the PHC and hospital settings. Our results revealed a high carriage rate of ESBL-EC in pregnant women ( $24.8 \%$ in PHC and $49.5 \%$ in hospitals) in Surabaya, Indonesia. The ESBL-E carriage rate was significantly higher in pregnant women in hospitals than in the PHC, along with a significantly higher rate of antibiotics use (19.8\% in the PHC and $41.4 \%$ in hospitals). The isolated ESBL-E strains were ESBL-EC (62 strains) and ESBL-KP (12 strains), and bla $a_{\mathrm{CTX}-\mathrm{M}-15}$ was the most prevalent (30.6\%) ESBL gene type. The clonal relatedness between PHC and hospital isolates was not found in this study. Continuous surveillance for ESBL-E carriage in pregnant women is strongly recommended to reduce the incidence of neonatal sepsis in Indonesia.

\section{Abbreviations}

ESBL

Extended-Spectrum Beta-Lactamase; ESBL-E:ESBL producing Enterobacteriaceae; ESBL-EC:ESBL producing E. coli; ESBLKP:ESBL producing Klebsiella pneumoniae; PHC:primary health care center; MRSA:methicillin-resistant Staphylococcus aureus; VLBW:very low birth weight; CLSI:Clinical and Laboratory Standards Institute; CAZ:ceftazidime; CTX:cefotaxime; FEP:cefepime; AMP:ampicillin; IPM:imipenem; MEPM:meropenem; AMK:amikacin; MI:minocycline; TE:tetracycline; ST:Trimethoprim-Sulfamethoxazole/co-trimoxazole; NA:nalidixic acid; CPFX:ciprofloxacine; CMZ:cefmetazole; FMOX:flomoxef; AZT:aztreonam; TZP:piperacillin-tazobactam; CL:colistin; TGC:tigecycline; PFGE:Pulsed-Field Gel Electrophoresis

\section{Declarations}

Ethics approval and consent to participate The study was approved by the Ethical Committees from the Dr. Soetomo Hospital (No. 0353/KEPK/VI/2018), Airlangga University Hospital (No. 193/KEH/2018), Kobe University Graduate School 
of Health Sciences (No. 393-1) and Kobe Tokiwa University (No. 19-11). In addition, all participants signed written informed consent for participation in the study.

Consent for publicationThis manuscript does not contain any individual person's data in any form.

Competing interestsNone to declare.

FundingThis study was supported by the Tahir Professorship of Airlangga University (grant No. 1149/UN3/2018), and the Japan Initiative for Global Research Network on Infectious Diseases (J-GRID) from the Japan Agency for Medical Research and Development (AMED) (Grant No. 18fm0108004h0004). (No. 1149/UN3/2018 to KK (kuntaman), and No. $18 \mathrm{fm} 0108004 \mathrm{~h} 0004$ to TS)

Authors' contributionsSROS, KO, TS and KK(Kuntaman) designed the study. SROS, MH, WS, HP and SK collected the samples and data, and performed laboratory testing. KK (Kitagawa), NN, RK and KO performed molecular analysis. SROS, TS and KK (Kuntaman) interpreted the data. SROS, TS and KK (Kuntaman) prepared for the first draft. IH, TS and KK(Kuntaman) revised and edited the final manuscript. All authors read and approved the final manuscript.

AcknowledgementsThe authors wish to acknowledge Mr. Gary Mawyer for his great support for proofreading.

Availability of data and materialsThe datasets during and/or analysed during the current study available from the corresponding author on reasonable request.

\section{References}

1. Paterson DL, Bonomo RA. Clinical Update Extended-Spectrum Beta-Lactamases: a Clinical Update. Clin Microbiol Rev [Internet]. 2005;18(4):657-86. Available from: http://www.ncbi.nlm.nih.gov/pmc/articles/PMC1265908/.

2. Denkel LA, Gastmeier P, Piening B. To screen or not to screen mothers of preterm infants for extended-spectrum betalactamase-producing Enterobacteriaceae (ESBL-E). J Perinatol. 2015;35(11):893-4.

3. Castillo-Tokumori F, Irey-Salgado C, Málaga G. Worrisome high frequency of extended-spectrum beta-lactamaseproducing Escherichia coli in community-acquired urinary tract infections: a case-control study. Int J Infect Dis. 2017;55:16-9.

4. Latifpour M, Gholipour A, Damavandi MS. Prevalence of extended-spectrum beta-lactamase-producing Klebsiella Pneumoniae isolates in nosocomial and community-acquired urinary tract infections. Jundishapur J Microbiol. 2016;9(3).

5. Goyal D, Dean N, Neill S, Jones P, Dascomb K. Risk factors for community-acquired extended-spectrum betalactamase-producing Enterobacteriaceae infections-a retrospective study of symptomatic urinary tract infections. Open Forum Infect Dis. 2019;6(2).

6. Kuntaman K, Santoso S, Wahjono H, Mertaniasih NM, Lestari ES, Farida H, et al. The Sensitivity Pattern of Extended Spectrum Beta Lactamase-Producing Bacteria Against Six Antibiotics that Routinely Used in Clinical Setting. J Indones Med Assoc. 2011;61(12):482-6.

7. KPRA-Kementerian-Kesehatan_[MoH RI]. Prevalensi dan pola kepekaan Escherichia coli and Klebsiella pneumoniae Penghasil Extended-spectrum Beta-Lactamase (ESBL) pada Isolat Klinik di Indonesia [Prevalence and its susceptibility pattern among ESBL producing E coli and Klebsiella pneumoniae] fro. 2016.

8. Imasari T, Tyasningsih W, Wasito EB, Kuntaman K. Prevalensi dan Pola Gen Extended Spectrum ? -lactamase Bakteri Usus Sapi Perah dan Penduduk Sekitar Peternakan di Surabaya. J Vet. 2018;19(3):1-10.

9. 10.1016/j.jiac.2020.01.010

Page 7/13 
Rosantia S, Higa T, Yagi N, Tokunaga T, Higa S, Yakabi Y, et al. Characterization of CTX-M-type-extended-spectrum beta-lactamase (ESBL)-producing Enterobacteriaceae isolated from Indonesian undergraduate medical students of a university in Surabaya, Indonesia. J Infect Chemother [Internet]. 2020;(xxxx). Available from:

https://doi.org/10.1016/j.jiac.2020.01.010.

10. Happy TA, Setyarini W, Ranuh IRG, Kuntaman K. Prevalence ESBL Producing Escherichia coli Among Children In Indonesia [Internet]. Universitas Airlangga; 2019. Available from: .

11. Abdel-Hady H, Hawas S, El-Daker M, El-Kady R. Extended-spectrum $\beta$-lactamase producing Klebsiella pneumoniae in neonatal intensive care unit. J Perinatol. 2008;28(10):685-90.

12. Pitout JDD. Infections with Extended-Spectrum $\beta$-Lactamase-Producing Enterobacteriaceae. Drugs. 2010;70(3):31333.

13. Rettedal S, Löhr IH, Bernhoff E, Natås OB, Sundsfjord A, Øymar K. Extended-spectrum $\beta$-lactamase-producing Enterobacteriaceae among pregnant women in Norway: Prevalence and maternal-neonatal transmission. J Perinatol. 2015;35(11):907-12.

14. Denkel LA, Schwab F, Kola A, Leistner R, Garten L, von Weizsäcker K, et al. The mother as most important risk factor for colonization of very low birth weight (VLBW) infants with extended-spectrum $\beta$-lactamase-producing Enterobacteriaceae (ESBL-E). J Antimicrob Chemother. 2014 Apr;69(8):2230-7.

15. Seale J, Millar M. Perinatal vertical transmission of antibiotic-resistant bacteria: A systematic review and proposed research strategy. BJOG An Int J Obstet Gynaecol. 2014;121(8):923-8.

16. Woerther PL, Burdet C, Chachaty E, Andremont A. Trends in human fecal carriage of extended-spectrum $\beta$ lactamases in the community: Toward the globalization of CTX-M. Clin Microbiol Rev. 2013;26(4):744-58.

17. Borer A, Gilad J, Menashe G, Peled N, Riesenberg K, Schlaeffer F. Extended-spectrum beta-lactamase-producing Enterobacteriaceae strains in community-acquired bacteremia in Southern Israel. Med Sci Monit [Internet]. 2002;8(1):CR44-7. Available from: http://www.ncbi.nlm.nih.gov/pubmed/11791130.

18. Barguigua A, Otmani F, El, Talmi M, Bourjilat F, Haouzane F, Zerouali K, et al. Characterization of extended-spectrum $\beta$-lactamase-producing Escherichia coli and Klebsiella pneumoniae isolates from the community in Morocco. J Med Microbiol. 2011;60(9):1344-52.

19. 10.1016/j.meegid.2018.04.005

Chong Y, Shimoda S, Shimono N. Current epidemiology, genetic evolution and clinical impact of extended-spectrum B-lactamase-producing Escherichia coli and Klebsiella pneumoniae. Infect Genet Evol [Internet]. 2018;61:185-8. Available from: https://doi.org/10.1016/j.meegid.2018.04.005.

20. 10.1016/j.ijid.2017.08.015

Bulabula ANH, Dramowski A, Mehtar S. Maternal colonization or infection with extended-spectrum beta-lactamaseproducing Enterobacteriaceae in Africa: A systematic review and meta-analysis. Int $\mathrm{J}$ Infect Dis [Internet]. 2017;64:58-66. Available from: https://doi.org/10.1016/j.ijid.2017.08.015.

21. Chereau F, Herindrainy P, Garin B, Huynh BT, Randrianirina F, Padget M, et al. Colonization of extended-spectrum- $\beta$ lactamase- and NDM-1-producing Enterobacteriaceae among pregnant women in the community in a low-income country: A potential reservoir for transmission of multiresistant Enterobacteriaceae to neonates. Antimicrob Agents Chemother. 2015;59(6):3652-5.

22. WANITA PERIPARTUM DI PUSKESMAS DAN RUMAH SAKIT Hasanah M. PERBANDINGAN PREVALENSI KARIER BAKTERI PENGHASIL ESBL PADA. WANITA PERIPARTUM DI PUSKESMAS DAN RUMAH SAKIT. 2019.

23. CLSI. Performance Standards for Antimicrobial Susceptibility Testing. 29th ed. CLSI supplement M100. Wayne, PA: Clinical and Laboratory Standards institute; 2019. 
24. Matsumura Y, Yamamoto M, Nagao M, Komori T, Fujita N, Hayashi A, et al. Multicenter retrospective study of cefmetazole and flomoxef for treatment of extended-spectrum- $\beta$-lactamase-producing Escherichia coli bacteremia. Antimicrob Agents Chemother. 2015;59(9):5107-13.

25. EUCAST. Testing Breakpoint tables for interpretation of MICs and zone diameters [Internet]. https://www.eucast.org/ast_of_bacteria/. 2020. p. 0-77. Available from: https://www.eucast.org/fileadmin/src/media/PDFs/EUCAST_files/Breakpoint_tables/v_10.0_Breakpoint_Tables.pdf. 26. 26.

27. Ueda S, Ngan BTK, Huong BTM, Hirai I, Tuyen LD, Yamamoto Y. Limited transmission of blaCTX-M-9-type-positive Escherichia coli between humans and poultry in Vietnam. Antimicrob Agents Chemother. 2015;59(6):3574-7.

28. Osawa K, Nakajima M, Kataoka N, Arakawa S, Kamidono S. Evaluation of antibacterial efficacy of drugs for urinary tract infections by genotyping based on pulsed-field gel electrophoresis (PFGE). J Infect Chemother. 2002;8(4):3537.

29. $10.1186 / \mathrm{s} 12859-015-0703-0$

Heras J, Domínguez C, Mata E, Pascual V, Lozano C, Torres C, et al. GelJ - a tool for analyzing DNA fingerprint gel images. BMC Bioinformatics [Internet]. 2015;16(1):1-8. Available from: http://dx.doi.org/10.1186/s12859-015-07030 .

30. Tenover FC, Arbeit RD, Goering RV, Mickelsen PA, Murray BE, Persing DH, et al. Interpreting chromosomal DNA restriction patterns produced by pulsed- field gel electrophoresis: Criteria for bacterial strain typing. J Clin Microbiol. 1995;33(9):2233-9.

31. Cantón R, Novais A, Valverde A, Machado E, Peixe L, Baquero F, et al. Prevalence and spread of extended-spectrum $\beta$ lactamase-producing Enterobacteriaceae in Europe. Clin Microbiol Infect. 2008;14(SUPPL. 1):144-53.

32. 10.1016/j.jmii.2013.05.006

Fan NC, Chen $\mathrm{HH}$, Chen CL, Ou LS, Lin TY, Tsai MH, et al. Rise of community-onset urinary tract infection caused by extended-spectrum $\beta$-lactamase-producing Escherichia coli in children. J Microbiol Immunol Infect [Internet]. 2014;47(5):399-405. Available from: http://dx.doi.org/10.1016/j.jmii.2013.05.006.

33. Fortini D, Fashae K, Villa L, Feudi C, García-Fernández A, Carattoli A. A novel plasmid carrying blaCTX-M-15 identified in commensal Escherichia coli from healthy pregnant women in Ibadan, Nigeria. J Glob Antimicrob Resist. 2015;3(1):9-12.

34. Klein EY, Van Boeckel TP, Martinez EM, Pant S, Gandra S, Levin SA, et al. Global increase and geographic convergence in antibiotic consumption between 2000 and 2015. Proc Natl Acad Sci U S A. 2018;115(15):E3463-70.

35. Kliebe C, Nies BA, Meyer JF, Tolxdorff-Neutzling RM, Wiedemann B. Evolution of plasmid-coded resistance to broadspectrum cephalosporins. Antimicrob Agents Chemother. 1985;28(2):302-7.

36. Doi Y, lovleva A, Bonomo RA. The ecology of extended-spectrum $\beta$-lactamases (ESBLs) in the developed world. J Travel Med. 2017;24(1):44-51.

37. Bauernfeind A, Stemplinger I, Jungwirth R, Ernst S, Casellas JM. Sequences of $\beta$-lactamase genes encoding CTX-M-1 (MEN-1) and CTX-M-2 and relationship of their amino acid sequences with those of other $\beta$-lactamases. Antimicrob Agents Chemother. 1996;40(2):509-13.

38. Bevan ER, Jones AM, Hawkey PM. Global epidemiology of CTX-M $\beta$-lactamases: Temporal and geographical shifts in genotype. J Antimicrob Chemother. 2017;72(8):2145-55.

39. Bui TMH, Hirai I, Ueda S, Bui TKN, Hamamoto K, Toyosato T, et al. Carriage of Escherichia coli producing CTX-M-type extended-spectrum $\beta$-lactamase in healthy Vietnamese individuals. Antimicrob Agents Chemother.

2015;59(10):6611-4. 


\section{Tables}

Table 1. Primer sets for PCR detection of ESBL-encoding genes

\begin{tabular}{|c|c|c|}
\hline Target & Primer name & Sequence $\left(5^{\prime}-3^{\prime}\right)$ \\
\hline \multirow[t]{2}{*}{ blaTEM } & T1 & CCGTGTCGCCCTTATTCC \\
\hline & $\mathrm{T} 2$ & AGGCACCTATCTCAGCGA \\
\hline \multirow[t]{2}{*}{ blaSHV } & S1 & ATTTGTCGCTTCTTTACTCGC \\
\hline & S2 & TTTATGGCGTTACCTTTGACC \\
\hline \multirow[t]{2}{*}{ blaCTX-M-1 group } & CTX-M-1-F & GCTGTTGTTAGGAAGTGTGC \\
\hline & CTX-M-1-R & CCATTGCCCGAGGTGAAG \\
\hline \multirow[t]{2}{*}{ blacTX-M-15 } & CTX-M-15S-F & ATGGTTAAAAAATCACTGCG \\
\hline & CTX-M-15S-R & TTACAAACCGTCGGTGACGA \\
\hline \multirow[t]{2}{*}{ blacTX-M-2 } & CTX-M-2S-F & ACGCTACCCCTGCTATTT \\
\hline & CTX-M-2S-R & сСТTTCCGССTTСTGСTC \\
\hline \multirow[t]{2}{*}{ blaCTX-M-9 group } & CTX-M-9-F & GCAGATAATACGCAGGTG \\
\hline & CTX-M-9-R & CGGCGTGGTGGTGTCTCT \\
\hline \multirow[t]{2}{*}{ blaCTX-M-14 } & CTX-M-14S-F & ATGGTGACAAAGAGAGTGCA \\
\hline & CTX-M-14S-R & TTACAGCCCTTCGGCGATGA \\
\hline
\end{tabular}

Table 2. Characteristics and ESBL-E carriage rates in the study population

\begin{tabular}{|clll|}
\hline & PHC (n=101) & Hospital (n=99) & Total (n=200) \\
\hline Age groups (Years) & & & \\
\hline $21>, n(\%)$ & $6(5.9 \%)$ & $5(5.1 \%)$ & $11(5.5 \%)$ \\
\hline $21-35, n(\%)$ & $82(81.2 \%)^{*}$ & $64(64.6 \%)$ & $146(73 \%)$ \\
\hline $35<, n(\%)$ & $13(12.9 \%)$ & $30(30.3 \%)^{*}$ & $43(21.5 \%)$ \\
\hline Antibiotics use, $n(\%)$ & $20(19.8 \%)$ & $41(41.4 \%)^{*}$ & $61(30.5 \%)$ \\
\hline ESBL-E carriage, $n(\%)$ & $25(24.8 \%)$ & $49(49.5 \%)^{*}$ & $74(37 \%)$ \\
\hline ESBL-EC, $n(\%)$ & $20(19.8 \%)$ & $42(42.4 \%)^{*}$ & $62(32 \%)$ \\
\hline ESBL-KP, n (\%) & $5(4.9 \%)$ & $7(7.1 \%)$ & $12(6 \%)$ \\
\hline
\end{tabular}

Table 3. Detected ESBL-encoding gene types in the isolated strains 


\begin{tabular}{|llll|}
\hline & PHC & Hospital & Total \\
\hline ESBL-EC, n & 20 & 42 & 62 \\
\hline CTX-M1, n (\%) & $1(5 \%)$ & $1(2.4 \%)$ & $2(3.2 \%)$ \\
\hline CTX-M14, n (\%) & $1(5 \%)$ & $2(4.8 \%)$ & $3(4.8 \%)$ \\
\hline CTX-M15, n (\%) & $11(55 \%) *$ & $8(19 \%)$ & $19(30.6 \%)$ \\
\hline CTX-M27, n (\%) & $1(5 \%)$ & $1(2,4 \%)$ & $2(3.2 \%)$ \\
\hline CTX-M55, n (\%) & $2(10 \%)$ & $4(9.5 \%)$ & $6(14.5 \%)$ \\
\hline TEM-1, n (\%) & $3(15 \%)$ & $7(16.7 \%)$ & $10(16.1 \%)$ \\
\hline ESBL-KP, n & 5 & 7 & 12 \\
\hline CTX-M15, n(\%) & $2(40 \%)$ & $4(57.1 \%)$ & $6(50 \%)$ \\
\hline CTX-M27, n (\%) & $0(0 \%)$ & $1(14.3 \%)$ & $1(8.3 \%)$ \\
\hline
\end{tabular}

chi-square test ${ }^{*} P<0.01$

Table 4. Phylogenetic groups of 62 strains of ESBL-EC

\begin{tabular}{|llll|}
\hline & PHC $(\mathbf{n = 2 0})$ & Hospital $(\mathbf{n = 4 2 )}$ & Total $(\mathbf{n = 6 2 )}$ \\
\hline A, n (\%) & $7(35 \%)$ & $12(28.6 \%)$ & $19(30.6 \%)$ \\
\hline B1, n (\%) & $4(20 \%)$ & $12(28.6 \%)$ & $16(25.8 \%)$ \\
\hline B2, n (\%) & $5(25 \%)$ & $6(14.3 \%)$ & $11(17.7 \%)$ \\
\hline D, n (\%) & $4(20 \%)$ & $12(28.6 \%)$ & $16(25.8 \%)$ \\
\hline
\end{tabular}

\section{Figures}




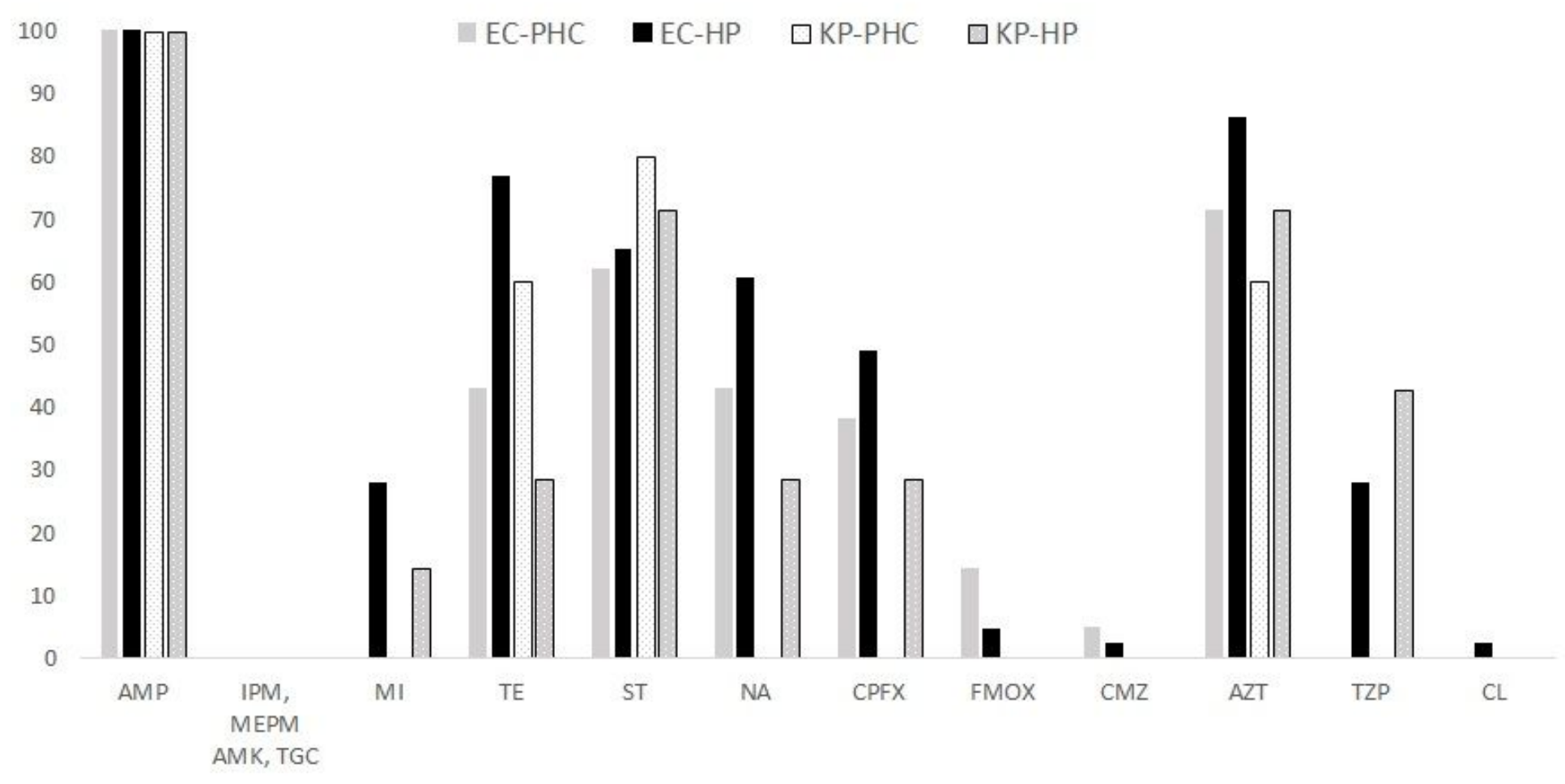

Figure 1

Percentage of antimicrobial resistance of ESBL-E from pregnant women in PHC and hospital $(\mathrm{n}=25)$ and hospitalized patients $(n=49)(X$ : antibiotic, Y: percentage of resistant isolates). EC-PHC: ESBL-EC in primary health care center $(n=20)$, EC-HP: ESBL-EC in hospital ( $n=42), K P-P H C$ : ESBL-KP in primary health care center $(n=5), K P-H P$ : ESBL-KP in hospital ( $n=7)$ AMP: ampicillin, IPM: imipenem, MEPM: meropenem, AMK amikacin, TGC: tigecycline, Ml: minocycline, TE: tetracycline, ST: Trimethoprim-Sulfamethoxazole/co-trimoxazole, NA: nalidixic acid, CPFX: ciprofloxacine, FMOX: flomoxef, CMZ: cefmetazole, AZT: aztreonam, TZP: piperacillin-tazobactam, CL: colistin

$\%$ Similarity

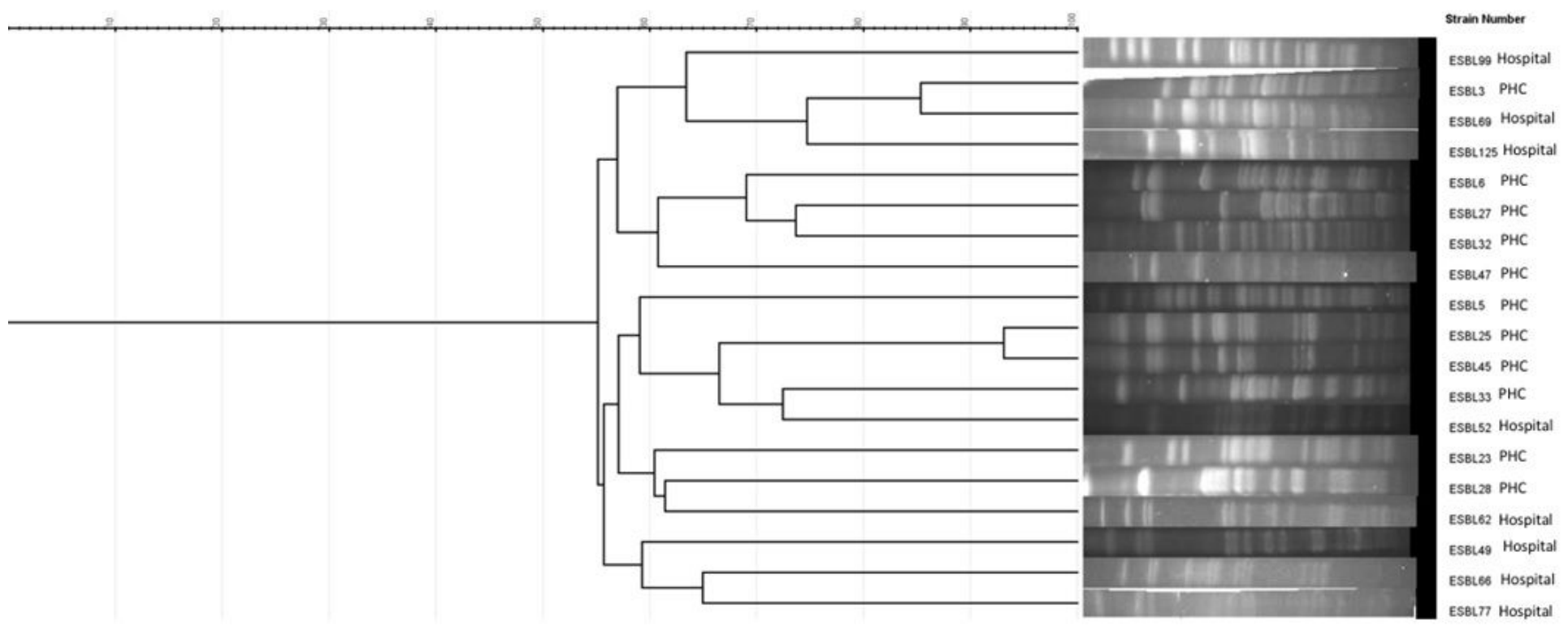

Figure 2 
The PFGE dendrogram of CTX-M-15 type ESBL-EC, isolates from rectal swab of pregnant women that visiting PHC (Primary Healthcare centre) and hospitals. CTX-M-15 type ESBL-EC isolates were subjected to Xbal-PFGE to evaluate whether there was clonal relatedness among those isolates. Identical (higher than $90 \%$ similarity ) Xbal-PFGE binding patterns were observed in two strains (ESBL-25 and 45) from pregnant women in PHC.

\section{Supplementary Files}

This is a list of supplementary files associated with this preprint. Click to download.

- OktasFigureoriginal.pptx 